\title{
Heterogeneity of luminal A and HER2-positive breast cancer based on DIA quantitative proteomics and bioinformatics analysis techniques
}

\section{Guogang Wu}

Ansteel Group Hospital https://orcid.org/0000-0001-9803-4425

\section{Lei Zhang}

China Medical University Hospital

Chunyan Liang

China Medical University Hospital

\section{Yao Cheng}

China Medical University Hospital

\section{Chenguang Lv}

China Medical University Hospital

\section{Bo Chen ( $\nabla$ chbyxl@163.com )}

Department of Breast Surgery, First Hospital of China Medical University, Shenyang, Liaoning Province, China https://orcid.org/0000-0002-2216-2794

\section{Primary research}

Keywords: Breast cancer, Heterogeneity, Proteomics, DIA, Bioinformatics analysis, IPA

Posted Date: May 20th, 2020

DOl: https://doi.org/10.21203/rs.3.rs-27419/v1

License: (c) (i) This work is licensed under a Creative Commons Attribution 4.0 International License. Read Full License 


\section{Abstract}

Background: Breast cancer has common tumor biological characteristics, but different characteristics also exist among different subtypes. Different treatment strategies have been adopted for different subtypes at present, but there are still many problems. Thus, an-depth study on the heterogeneity of different types of breast cancer will help to identify new diagnostic therapeutic targets and solve the existing problems of individualized treatment for breast cancer.

Methods: In this study proteins of HER2-positive breast cancer, luminal A breast cancer, and para-cancer tissues were quantified based on data independent acquisition (DIA) proteomics technology, and the differentially expressed proteins (DEPs) were screened and analyzed by IPA software and the database among the luminal A (LA) versus para-cancer tissues (PT) and HER2 versus LA groups.

Results: There were 264 up- and 123 down-regulated proteins in the LA versus PT groups. PD- 1 and PDL1 cancer immunotherapy pathways were significantly inhibited, and XBP1 was predicted to be the strongest activator. MTOR was predicted to be the strongest inhibitor. The DEPs were significantly associated with "Cancer" and "Organismal Injury and Abnormalities." There were 134 up- and 286 downregulated proteins in the HER2 versus LA groups. Signaling by rho family GTPases was significantly inhibited. XBP1 was predicted to be the strongest activator. TGFB1 was predicted to be the strongest inhibitor. The DEPs were significantly associated with "Cancer," "Endocrine System Disorders," and "Organismal Injury and Abnormalities."

Conclusion: Luminal A breast cancer may be insensitive to PD-1 and PD-L1 inhibitors. Signaling by rho family GTPases and RhoGDI signaling play a unique role in the proliferation and metastasis of luminal A and HER2-positive breast cancer, respectively. XBP1 is a promising new target for the treatment of breast cancer, and TGFB1 may play different biological roles in HER2-positive and luminal A breast cancer.

\section{Background}

Breast cancer is the most frequent malignancy in women and a heterogeneous disease at the molecular level ${ }^{[1]}$. The identification of different molecular subtypes occurs through the use of techniques, including immunohistochemistry ${ }^{[2]}$ and gene expression profiling ${ }^{[3]}$. Different molecular subtypes of breast cancer not only have some common characteristics of malignant tumors, but also different biological features ${ }^{[4-}$ 6]. For example, the luminal A subtype of breast cancer has positive estrogen receptor (ER) and/or progesterone receptor (PR), a Ki67 $<20 \%$, and negative human epidermal growth factor receptor (HER2) ${ }^{[7]}$, which is generally associated with a highly favorable prognosis ${ }^{[8]}$ and typically exhibits less frequent and less extensive lymph node involvement ${ }^{[9-10]}$. The luminal A subtype of breast cancer also tends to have a more indolent course with a slower evolution over time when compared with the other molecular subtypes ${ }^{[11]}$. The HER2-positive (non-luminal) subtype of breast cancer is ER- and PR-negative, and HER2-positive ${ }^{[7]}$, which is generally associated with an unfavorable prognosis. HER2 overexpression is associated with a higher frequency of locoregional recurrence and metastasis (ranging between $4 \%$ and 
$15 \%$ ) based on studies in which patients were not treated with HER2-targeted therapy ${ }^{[4,12-14]}$. An in-depth study on the heterogeneity of different molecular subtypes of breast cancer will have important clinical value for individualized treatment of breast cancer. Proteins play an essential role in the biological processes associated with cancer. The altered levels of protein expression can deregulate critical cellular pathways and interactive networks, which will affect the biological cellular functions and compromise the tissue environment homeostasis, thus contributing to cancer development ${ }^{[15]}$. A comprehensive and systematic discovery of proteomic differences in different subtypes of breast cancer will help to identify new diagnostic and therapeutic targets.

The quantitative proteomics methods have been used to identify the protein content of tissues under specific physiologic conditions based on data independent acquisition (DIA) technology and has undergone rapid development in recent years. DIA technology combines a traditional proteomics "shotgun method" and mass spectrum quantitative "gold standard" absolute choice reaction monitoring/multiple reaction monitoring (SRM/MRM) advantages and characteristics of technology. Further, there are no omissions, no difference in acquiring all the ions in the samples of all pieces of information, and protein quantification is more accurate. This method will be more helpful in detecting differentially expressed proteins (DEPs) among different subtypes of breast cancer, especially the subtypes missed in the previous screening, so that we can have a more comprehensive understanding of the DEPs information.

Bioinformatics is an essential mainstream technology of proteomics research. Ingenuity pathways analysis (IPA) used in this study is currently the most abundant and annotated biochemical analysis software and database ${ }^{[16]}$. IPA is a proof-of-knowledge based software that helps researchers to model $\mathbb{}$ analyze, and understand the complex biological and chemical systems in life science research. IPA has an extensive repository of biological and chemical knowledge, thus offering the researcher access to the most current findings available on genes, protein families, normal cellular and disease processes, and signaling pathways. The software facilitates looking for information on genes and proteins, the impact of genes and proteins on diseases and cellular processes, and the role of genes and proteins in signaling pathways ${ }^{[17]}$.

In this study we used DIA proteomics technology integrated with IPA software and database to investigate genes, signaling pathways, upstream regulators, and regulatory effects that could be associated with DEPs among luminal A (LA) breast cancer and para-cancer tissues (PT) samples (LA versus PT) , HER2-positive subtype breast cancer and LA breast cancer samples (HER2 versus LA).

\section{Methods}

\section{Breast cancer sample collection}

In our study, 52 human breast cancer tissue specimens and 3 para-cancer tissue samples were collected from breast cancer patients who underwent surgical resections in China Medical University. (Shenyang, China). Para-cancer tissue is non-cancerous tissue, derived from normal breast tissue $2 \mathrm{~cm}$ from the 
margin of luminal A breast cancer. All tissue specimens were retained for pathologic examination with $\mathrm{HE}$ staining to determine the pathologic type and other auxiliary tests, such as immunohistochemical and FISH testing. The remaining tissue specimens were washed with cold phosphate buffered saline (GE Healthcare, Beijing, China) to remove the residual blood after surgical resection, then immediately frozen in liquid nitrogen and stored at $-80^{\circ} \mathrm{C}$ for further analysis. None of the 52 breast cancer patients received pre-operative radiation, chemotherapy, or other treatments. This study was conducted in accordance with the principles expressed in the Helsinki declaration and approved by the Research Ethics Committee of China Medical University. (IRB approval number:AF-SOP-07-1.1-01). Informed consent was obtained from all patients. A summary of clinical and pathologic data for the breast cancer patients is shown in Supplementary Table 1 (blue data represent 3 tissue adjacent to carcinoma samples $₫$ green data represent 3 luminal A breast cancer samples and red data represent 3 HER2(+) samples).

\section{Experimental procedures and strategies}

Fifty-five samples were established by Data Dependent Acquisition(DDA) mass spectrometry. Then, based on the constructed database, DIA relative quantitative proteomics analysis was performed on 3 HER2 positive breast cancer samples, 3 luminal A breast cancer samples and 3 para-cancer tissues samples to compare the differences among LA vs PT, HER2 vs LA and screen out all the DEPs. After that, we continued to perform IPA-based bioinformatics analysis of the DEPS.

\section{Sample preparation}

The tissue was scraped and added with an appropriate amount of SDT lysate, then transferred to a Lysing Matrix A tube. The homogenizer was used for homogenization crushing $(24 \times 2,6.0 \mathrm{~m} / \mathrm{S}, 60 \mathrm{~s}$, twice). After ultrasound, the homogenized tissue was placed in a boiling water bath for $10 \mathrm{~min}$, then centrifuged at $14000 \mathrm{~g}$ for $15 \mathrm{~min}$. The supernatant was filtered through a $0.22-\mathrm{m}$ centrifuge tube and the filtrate was collected. Protein was quantified using the BCA method. The samples were packed and stored at $-80^{\circ} \mathrm{C}$.

\section{SDS-PAGE electrophoresis}

Protein $(20 \mu \mathrm{g})$ was taken from each sample and added to a $6 \mathrm{X}$ sample loading buffer, followed by a boiling water bath for $5 \mathrm{~min}$, separation on 12\% SDS-PAGE (constant pressure 250V for $40 \mathrm{~min}$ ), and staining with Coomassie brilliant blue.

\section{FASP enzyme ${ }^{[18]}$}

A 200-ug protein solution was taken from each sample. DTT was added to a final concentration of $100 \mathrm{mM}$ and the solution was placed in a boiling water bath for $5 \mathrm{~min}$, then the samples were cooled to room temperature. Two hundred micron of UA buffer was added to the protein solution and mixed well, then transferred to a $30 \mathrm{kD}$ ultrafiltration centrifuge tube and centrifuged at $12,500 \mathrm{~g}$ for $25 \mathrm{~min}$. The filtrate was discarded (this step was repeated twice). One hundred microliters of IAA buffer in UA was 
added, oscillated at $600 \mathrm{rpm}$ for $1 \mathrm{~min}$, reacted at room temperature against light for $30 \mathrm{~min}$, and centrifuged at 12,500 g for $25 \mathrm{~min}$. One hundred microliters of UA buffer was added and centrifuged at $12,500 \mathrm{~g}$ for $15 \mathrm{~min}$ (this step was repeated twice). One hundred microliters of $0.1 \mathrm{M}$ TEAB solution was added and centrifuged at $12,500 \mathrm{~g}$ for $15 \mathrm{~min}$ (this step was repeated twice). Forty microliters of trypsin buffer ( $4 \mu \mathrm{L} g$ of trypsin in $40 \mathrm{mu} \mathrm{L}$ of $0.1 \mathrm{M}$ TEAB solution) was oscillated at $600 \mathrm{rpm}$ for $1 \mathrm{~min}$, and placed at $37^{\circ} \mathrm{C}$ for $16-18 \mathrm{~h}$. The collection tube was replaced, centrifuged at $12,500 \mathrm{~g}$ for $15 \mathrm{~min}, 20 \mu \mathrm{L}$ of $0.1 \mathrm{M}$ TEAB solution was added, centrifuged at $12500 \mathrm{~g}$ for $15 \mathrm{~min}$, and the filtrate was collected. The peptide fragment was desalted with a C 18 cartridge, freeze-dried, and re-dissolved with $40 \mu \mathrm{L}$ of $0.1 \%$ formic acid solution. The peptide fragment was quantified.

\section{High PH RP classification}

All the peptide compounds were classified using an Agilent 1260 Infinity II HPLC system. Buffer solution A was $10 \mathrm{mM} \mathrm{HCOONH}_{4}$ and $5 \%$ can (pH 10.0) and buffer solution $\mathrm{B}$ was $10 \mathrm{mM} \mathrm{HCOONH}_{4}$ and $85 \%$ can (pH 10.0). The column was balanced with liquid $A$ and the samples were separated from the column (Waters, XBridge Peptide BEH C18 Column, 130A, $5 \mathrm{~m}, 4.6 \mathrm{~mm}$ X $100 \mathrm{~mm}$ ) with a flow rate of $1 \mathrm{~mL} / \mathrm{min}$. The liquid phase gradient was as follows: using a linear gradient, the column temperature was maintained at $30^{\circ} \mathrm{C}$ within 40 min from $5 \%$ B to $45 \%$ B. Thirty-six components were collected and dried in a vacuum concentrator. After freeze-drying, the samples were re-dissolved with $0.1 \%$ formic acid solution in 12 fractions.

\section{DDA mass spectrometry library}

Nine microliters were taken from each fraction and added to $1 \mathrm{ul}$ of 10x iRT peptide fragment. After mixing, 6 ul of sample was injected, separated by nano-lc, and analyzed by on-line electrospray tandem mass spectrometry. The whole liquid-mass series system was as follows: 1) liquid phase system, Easy nLC system (Thermo Fisher Scientific); 2) mass spectrometry system, q-exactive hf- $x$ (Thermo Fisher Scientific). Buffer solution A was $0.1 \%$ formic acid solution and solution B was $0.1 \%$ acetonitrile solution (acetonitrile was $80 \%$ ). The samples were separated by a gradient of non-linear growth in an analytical column (Thermo Fisher Scientific, Acclaim PepMap RSLC 50 um X $15 \mathrm{~cm}$, nano viper, P/N164943) at a flow rate of $300 \mathrm{~nL} / \mathrm{min}$ for $0-5 \mathrm{~min}, 1 \% \mathrm{~B}$ for $5-95 \mathrm{~min}, 1 \% \mathrm{~B}$ to $28 \% \mathrm{~B}$ for $95-110 \mathrm{~min}, 28 \%$ B to $38 \%$ B for $110-115 \mathrm{~min}, 38 \% \mathrm{~B}$ to $100 \% \mathrm{~B}$ for $115-120 \mathrm{~min}$, and $100 \% \mathrm{~B}$. The electrospray voltage was $2.0 \mathrm{kV}$.

The mass spectrum parameters were set as follows: (1) MS, scan range $(\mathrm{m} / \mathrm{z})=350-1500$; resolution=60,000; $\mathrm{AGC}$ target $=3 \mathrm{e} 6$; maximum injection time $=30 \mathrm{~ms}$; included charge states $=2-7$; filter dynamic exclusion: exclusion duration $=30 \mathrm{~s}$; and (2) dd-MS2, isolation window $=1.6 \mathrm{~m} / \mathrm{z}$; resolution=15,000; $A G C$ target=1e5; maximum injection time $=45 \mathrm{~ms}$; and NCE=28\%.

The Spectronaut Pulsar X (version 12, Biognosys AG) was used to consolidate and analyze the original mass spectrometry data and establish a spectrograph database. The database was Swissprot_human_isoform_201806 (42356 entries), as below: 2018-06, download link: http://www.uniprot.org. Trypsin enzymolysis was set to allow two missing cutting sites. 
Carbamidomethyl (C), variable modification: Oxidation (M), and acetyl (Protein n-term) n-terminal acetylation. The standard for database construction was $1 \%$ precursor false discovery rate( FDR), $1 \%$ protein FDR, and $1 \%$ peptide FDR.

\section{DIA mass spectrometry}

Nine samples were removed and $6 \mathrm{ul}$ was added to $1 \mathrm{ul}$ of 10x iRT peptide, mixed, separated with nano-lc, and analyzed by on-line electrospray tandem mass spectrometry. The whole liquid-mass series system was as follows: 1) liquid phase system, Easy nLC system (Thermo Fisher Scientific); and 2) mass spectrometry system, q-exactive hf-x (Thermo Fisher Scientific). Buffer solution A was $0.1 \%$ formic acid solution and solution B was $0.1 \%$ acetonitrile solution (acetonitrile was $80 \%$ ). The samples were separated by a gradient of non-linear growth in the analytical column (Thermo Fisher Scientific, Acclaim PepMap RSLC 50um X 15cm,nano viper, P/N164943) at a flow rate of $300 \mathrm{~nL} / \mathrm{min}$ for $0-5 \mathrm{~min}, 1 \% \mathrm{~B}$ for 5$95 \mathrm{~min}, 1 \% \mathrm{~B}$ to $28 \% \mathrm{~B}$ for $95-110 \mathrm{~min}, 28 \% \mathrm{~B}$ to $38 \%$ B for $110-115 \mathrm{~min}, 38 \% \mathrm{~B}$ to $100 \%$ B for $115-120$ min, and $100 \% \mathrm{~B}$. The electrospray voltage was $2.0 \mathrm{kV}$.

The mass spectrum parameters were set as follows: (1) MS, scan range $(\mathrm{m} / \mathrm{z})=350-1500$; resolution=60,000; AGC target=3e6; maximum injection time=50 ms; and (2) DIA, resolution=15,000; AGC target $=2 \mathrm{e} 5$; maximum injection time $=45 \mathrm{~ms}$; and $\mathrm{NCE}=28 \%$.

\section{Bioinformatics Analyses}

This research use the IPA data Analysis software and database. The DEPs performed IPA-based classical pathway analysis, upstream regulation analysis, disease and function analysis, and regulation effect analysis. A detailed description of these analyses is provided in the supplementary information.

\section{Results}

\section{Protein identification}

A total of 149,700 precursors $₫ 113,117$ peptides, and 9730 protein groups were detected in 55 samples. The constructed database was used for qualitative and quantitative analyses of proteins in all samples, among which the quantitative results of LA breast cancer and PT proteins are listed in Supplementary Table 2, and the quantitative results of HER2 $(+)$ and LA breast cancer proteins are listed in Supplementary Table 3. Each comparison of the parallel between the two groups of samples was better (Figure 1a,1b) and the data had a significant difference (Figure 1c,1d). Therefore, we chose two groups with at least one-half of the data as a null value with a significant difference through the data analysis, screening protein with fold-change values $\geq 1.5$ and $\leq-1.5$, and a $P$ value $<0.05$ as the DEPSs. The results in the LA versus PT groups comparison indicated that 264 proteins were up-regulated and 123 proteins were down-regulated, for a total of 387 DEPs. Osteomodulin (OMD) was the up-regulated protein observed with the highest fold-change value ( $\mathrm{FC}=16.98425, \mathrm{P}=0.028912$; Supplementary Table 2 [red data represent the up-regulated proteins and the green data represent the down-regulated proteins]). In the 
HER2 versus LA groups comparison, 134 proteins were up-regulated and 286 proteins were downregulated, for a total of 420 DEPs. Nucleoporin NUP188 homolog (NUP188) was the up-regulated protein observed with the highest fold-change $(F C=20.17439, P=0.000299$; Supplementary Table 3 [red data represent the up-regulated proteins and green data represent the down-regulated proteins]). Among the two comparison groups, 7 DEPs presented a gradual increase in the expression from the PTs to the HER2positive breast cancer samples, with the highest levels observed in the HER2-positive breast cancer

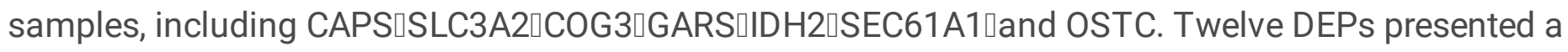
gradual decrease in expression, with the lowest levels observed in the HER2-positive breast cancer

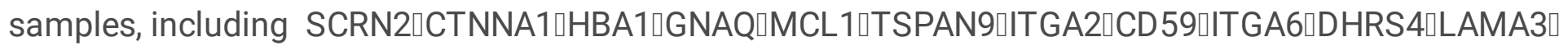
and RGL2.

\section{Bioinformatics analysis based on IPA}

\section{Classic pathway analysis}

In the LA versus PT groups comparison, IPA analysis identified a total of 400 signaling pathways were associated with the DEPs (Supplementary Table 4). The neuroinflammation signaling and dendritic cell maturation and oxidative phosphorylation pathways were significantly activated (- $\log [p$ value] $->1.31, z$ - score $\geq 2$ ), and the PD-1 and PD-L1 cancer immunotherapy pathways were significantly inhibited (-log [p-value] $>1.31, z$-score $\leq-2$; Figure 2a). We further analyzed the trend change of the DEPs in the inhibited PD-1 and PD-L1 cancer immunotherapy pathways (-log [p-value] $=2.08, z$-score $=-2.449)$, and found that the $\mathrm{MHC1}, \mathrm{MHC} 1, \mathrm{MHC}$, and $\mathrm{CK} 2$ molecules in the pathway were significantly up-regulated and PDCD4 was significantly down-regulated (Figure 3a). According to previous reports, CK2 and other molecules of $\mathrm{MHC1}, \mathrm{MHC} 1$, and $\mathrm{MHC} 2$ were significantly down-regulated and PDCD4 was significantly up-regulated

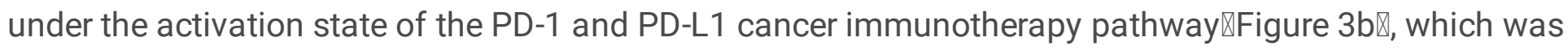
consistent with the results of the current study.

In the HER2 versus LA group comparison, IPA analysis identified a total of 437 signaling pathways were associated with the DEPs (Supplementary Table 5). The RhoGDI signaling pathway was significantly activated (-log [p-value] $>1.31, z$-score $\geq 2$ ); signaling by the Rho family GTPases and other 38 signaling pathways were significantly inhibited (-log [p-value] $>1.31$, z-score $\leq-2^{\prime}$ Figure $2 b$ ). We further analyzed the change in trend of DEPs in the inhibited state of signaling by the Rho family GTPases pathway (-log [p-value] $=7.38, z$-score $=-2.324)$. We found that the NADPH xxidase, ARP2/3, Cofilm, and other

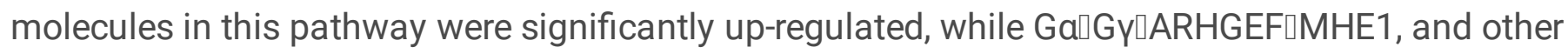
molecules were significantly down-regulated (Figure 3c), which was consistent with the above molecular change in trend reported in previous studies under the activation state of signaling by Rho family GTPases (Figure 3d), except ARP2/3 and Cofilm.

In addition, there were three inhibitory pathways among the two groups comparison (leukocyte extravasation signaling冈agrin interactions at neuromuscular junction『and protein kinase A signaling).

\section{Upstream regulation analysis}


In the LA versus PT groups comparison, IPA analysis identified a total of 768 regulatory factors that may play a role in the DEPs (Supplementary Table 6) and predicted 46 strong activators (score $\geq 2$ ), such as XBP1, ERN1, EDN1, and IRF2. XBP1 was predicted to be the strongest activator (score $=3.159$ ), and there were 21 genes that were uniformly activated (Figure 5a). MTOR, MYCN, CCN5, NKX2-3, and 14 other molecules were predicted to be strong inhibitory factors (score $\leq-2$ ). MTOR was predicted to be the strongest inhibitory factor (score $=-2.785)$

In the HER2 vs LA groups' comparison, IPA's analysis identified a total of 533 regulatory factors may play a role in the DEPs (Supplementary Table 7)and predicted 15 strong activators (score $\geq 2$ ), such as XBP1, ERN1, EDN1 and IRF2.Among them,XBP1 was predicted to be the strongest activator (score= 2.557).Meanwhile, 24 strong inhibitory factors, such as TGFB1, were predicted (score $\leq-2)$, and TGFB1 was the strongest inhibitory factor (score= -3.236 ).

In addition, five activators were the same among the two groups comparison (XBP1, ATF6, IFNG, EBI3, and interferon-alpha) para-cancer tissues and one same activator (NKX2-3).

\section{Disease and function analysis}

The IPA "disease and function" analysis revealed that DEPs in the LA versus PT groups comparison were significantly associated with "Cancer" and "Organismal Injury and Abnormalities" (Figure 4a), and there was activation or inhibition relationship with disease and function (Figure 5a). The significant activation of diseases or functions included "Infection of tumor cell lines" (z-score $=4.019)$ and "Infection by RNA Virus" (z-score $=3.797)$. Significant inhibition of diseases or functions included "Cell Death of Tumor Cells" (z-score $=-2.433)$ and "Gastrointestinal Tract Cancer" (z-score $=-2.401$; Supplementary Table 8).

At the same time, we also found that DEPs in the HER2 versus LA groups comparison were significantly associated with "Cancer," "Endocrine System Disorders," and "Organismal Injury and Abnormalities" (Figure $4 \mathrm{~b}$ ) and there was an activating or inhibitory relationship between disease and function (Figure $5 b)$. Significant activation of diseases or functions included "Morbidity or Mortality" (z-score $=4.522$ ) and "Organismal Death" (z-score $=4.369)$. Significant inhibition of diseases or functions included "Invasion of Cells" (z-score =-4.090) and "Cell Spreading" (z-score =-3.437; Supplementary Table 9).

\section{Regulation effect analysis}

In this study, DEPs were involved in the upstream regulatory network and the downstream function of the possible pathways of action. The networks of LA versus PT groups comparison are shown in Supplementary Table 10 and the networks of HER2 versus LA groups comparison are shown in Supplementary Table 11. The Consistency Score is a measure of the consistency and density of causality among upstream regulatory factors, data sets, and disease and function in the network. The higher the consistency score, the more accurate the results of regulatory effects. We chose the first regulatory network in the analysis of regulatory effects. In the LA versus PT groups comparison, the network due to the following regulators were able to activate "Cell Movement of Kidney Cell Lines," "Cell Spreading," and 
"Invasion of Carcinoma Cell Lines" (Figure 6a). In the HER2 versus LA groups comparison, the network due to the following regulators were able to activate "Organismal Death," "Motor Dysfunction or Movement Disorder," and inhibit "Cell Spreading," "Interaction of Tumor Cell Lines," "Migration of Tumor Cell Lines," "Organization of Cytoplasm," and "Shape Change of Tumor Cell Lines" (Figure 6b).

\section{Discussion}

The various subtypes of breast cancer have the same biological characteristics of malignancy, exhibit heterogeneity, and determine different treatment methods and prognosis. Comparing detailed HER2positive breast cancer囚LA breast cancer, and PT proteomics differences will provide more biological information of breast cancer subtypes, contribute to a clearer understanding of the heterogeneity of HER2-positive and LA breast cancer, and provide references for screening targets and guiding individualized treatment.

We first established a breast cancer mass spectrum library by DDA, including various subtypes of breast cancer samples. The purpose was to acquire comprehensive proteomics information about breast cancer, provide a background reference for the quantitative analysis of DIA technical proteins in HER2-positive and LA breast cancer subtypes, and realize the deep coverage and accurate quantitative analysis of proteins in low abundance. In addition, the mass spectrometry library we built permanently stored the data for subsequent retrospective analysis.

We identified some DEPs among the groups comparison, which lay the foundation for our future target screening. OMD was the most significantly up-regulated protein observed in the LA versus PT groups comparison. $\mathrm{OMD}$ is a cell-binding keratin sulphate proteoglycan that belongs to the family of leucinerich repeat proteins found in the mineralized matrix of bone and is primarily expressed by mature osteoblasts. Breast cancer preferentially metastasizes to the bone primarily forming osteolytic lesions characterized by loss of bone density [19] [20]. Relevant studies have shown that the incidence of bone metastasis in LA breast cancer patients is significantly higher than other subtypes [21]. OMD has been proposed to be involved in the regulation of cell proliferation and migration, and therefore this may of particular interest in breast cancer progression to bone metastasis ${ }^{[22]}$. OMD might therefore provide a potentially novel new biomarker for LA breast cancer progression and clinical outcome.

The PD-1 / PD-L1 checkpoint and its inhibitors have been the focus of research in tumor immunology in recent years ${ }^{[23]}$. The theoretical basis is the immune escape mechanism of tumors ${ }^{[24-25]}$. Tumor and immune cells activate the PD-1 / PD-L1 checkpoint and up-regulate the expression of PD-L1, which can negatively regulate the anti-tumor immune response ${ }^{[26]}$. Current studies have shown that expression in PD-L1 is up-regulated in triple negative and HER-2 overexpressed breast cancer, which has a high predictive value for prognosis ${ }^{[27-32]}$. PD-1 / PD-L1 inhibitor has become an effective treatment for melanoma ${ }^{[33]}$, non-small cell lung cancer ${ }^{[34]}$, Hodgkin lymphoma ${ }^{[35]}$, hepatocellular carcinoma ${ }^{[36]}$, and other solid tumors. We performed IPA-based classic pathway analysis of the DEPs in the LA versus PT groups comparison and showed that PD-L1 expression was not up-regulated and PD-1 and PD-L1 cancer 
immunotherapy pathways were significantly inhibited. We speculated that LA breast cancer may have another characteristic that is insensitive to PD-1 / PD-L1 inhibitors, similar to insensitivity to chemotherapy.

The Rho family of GTPases plays a key role in regulating a range of biological activities, including actin tissue, focal complex/adhesion assembly, cellular activity, cell polarity, gene transcription, and cell cycle progression. Some Rho GTPases and their signaling elements are overexpressed or overactive in breast cancer. Rho GTPases promote cell cycle and reduce intercellular adhesion, playing an important role in breast cancer cell metastasis. Numerous studies have shown that the Rho GTPase signaling pathway affects the proliferation and metastasis of breast cancer cells in vivo and in vitro ${ }^{[370}$. We found that signaling by Rho family GTPases in the HER2 versus LA groups comparison were significantly inhibited, but it was activated in the LA versus PT groups comparison. We speculated that this signaling pathway may play a unique role in the proliferation and metastasis of LA breast cancer. We also showed that RhoGDI signaling was significantly activated in the HER2 versus LA groups comparison, but it was inhibited in the LA versus PT groups comparison. RhoGDI (RhoGTP-dissociation inhibitor) is considered to be a regulatory factor of Rho GTPases, which has been shown to be highly expressed in metastatic liver ${ }^{[38]}$ and colon cancers ${ }^{[39]}$, and was once considered a predictor of distant metastasis of tumors. High expression of RhoGDI can enhance the activity of PI3K/AKT and MAPK pathways, stimulate the growth and metastasis of tumor cells, and play an important role in the occurrence and development of tumors ${ }^{[40]}$. Therefore, we speculated that RhoGDI signaling pathway also play a specific and important role in the proliferation and metastasis of HER2-positive breast cancer. RhoGDI signaling may provide targets for HER2-positive breast cancer therapy.

$\mathrm{X}$-box binding protein 1 (XBP1) is the main regulator of endoplasmic reticulum stress (ERS) with basic lucien zipper structure ${ }^{[41][42]}$. A large number of studies have shown that XBP1 is highly expressed in a variety of malignant tumor cells, and can promote the hypoxic survival of tumor cells and induce tumor metastasis and drug resistance ${ }^{[43]}$. XBP1 can also induce tumor angiogenesis and inhibit tumor-related immunity through a variety of mechanisms, which lead to the rapid proliferation and immune escape of malignant tumor cells, and thus accelerate the invasion and metastasis of malignant tumors [43] [44]. When we performed IPA-based upstream regulatory factor analysis, we showed that XBP1 was predicted to be the strongest activator in both groups comparison. Therefore, XBP1 is a highly promising target for the treatment of LA and HER2-positive breast cancer. In addition, we identified a common inhibitor of ATF6, IFNG, EBI3, interferon-alpha, and a common activator of NKX2-3, which are also potential new targets for the treatment of breast cancer.

Transforming growth factor-beta (TGF- $\beta$ ) is a polypeptide growth factor with various biological activities and is widely distributed in various tissues. In mammals, TGF- $\beta$ has three isoforms (TGF- $\beta 1,2$, and 3 , of which TGF- $\beta 1$ is most abundant in the TGF- $\beta$ family ${ }^{[45]}$. TGF- $\beta 1$ is closely related to the occurrence and development of tumors ${ }^{[46]}$. TGF- $\beta 1$ is thought to have a dual role in breast cancer ${ }^{[47]}$. TGF- $\beta 1$ initially acts as an anti-cancer agent in breast cancer progression. In the whole process of the development of 
breast cancer, however, cells on the proliferation of TGF- $\beta 1$ became more and more resistant, and with the evolution of the tumor, TGF- $\beta 1$ lost inhibition for the growth of cancer cells and promoted the progress of the tumor, partly through enhanced tumor cell motility and invasiveness and the capacity to form metastases ${ }^{[48-51]}$. In our upstream regulatory analysis in the HER2 versus LA groups comparison, we predicted that TGF- $\beta 1$ was predicted to be the strongest inhibitory factor. Therefore, we speculated that TGF- $\beta 1$ may also play different roles in HER2-positive and LA breast cancer; the specific mechanism will be the subject of a corollary study.

We performed IPA-based upstream regulatory factor analysis of the DEPs in the LA versus PT groups comparison. The mechanistic target of rapamycin (MTOR) was predicted to be the strongest inhibitor. MTOR plays an important role in cell proliferation, differentiation, metastasis, and survival. MTOR has become a new target in cancer therapy ${ }^{[52]}$. Currently, MTOR inhibitors have been extensively studied in various cancers ${ }^{[53]}$, and we expect good results. In addition, other strong activating or inhibitory factors also play different roles in various subtypes of breast cancer, which is also worthy of attention.

Finally, we found that the DEPs of the two groups comparison were relatively similar in disease and function analysis, mainly enriched in "Cancer" and "Organismal Injury and Abnormalities", and proved the value of the DEPs in this study. It was also found that DEPs in the HER2 versus LA groups comparison were also enriched in "Endocrine System Disorders," which is consistent with our previous understanding that LA breast cancer is an estrogen-dependent tumor. We have also identified some diseases and functions that are closely related to the DEPs that produce activating or inhibitory effects. Some of the factors are closely associated with breast cancer. We created the control effect of network diagram, which enabled us to deepen the understanding of these diseases and functions, and the main control factors, such as CDH1, ETV4, MTOR, NOTCH3, ERG, MIR - b - 3 p, 29 MKNK1, and SPDEF. It is of great value for us to further study the biological characteristics of different subtypes of breast cancer.

\section{Conclusions}

Based on DIA technology and IPA bioinformatics analysis, we obtained heterogeneity information of different subtypes of breast cancer, and found that LA breast cancer may be insensitive to PD-1/PD-L1 inhibitors. Signaling by Rho family GTPases and RhoGDI signaling play a unique role in the proliferation and metastasis of LA and HER2-positive breast cancer, respectively. XBP1 is a promising new target for the treatment of breast cancer, and TGF- $\beta 1$ may play different biological roles in HER2-positive and LA breast cancer. These findings are of great value for the individualized study of different subtypes of breast cancer in the future.

\section{Abbreviations}

HER2:Human epidermal growth factor receptor

DEPs:Differentially expressed proteins

Page $11 / 23$ 
DIA: Data-independent acquisition

IPA:Ingenuity Pathways Analysis

OMD:Osteomodulin

NUP188:Nucleoporin NUP188 homolog

XBP1:X - box binding protein 1

TGFB1: Transforming growth factor $\beta 1$

MTOR: Mechanistic target of rapamycin

\section{Declarations}

\section{Ethics approval and consent to participate}

This study was approved by the Research Ethics Committee of China Medical University. (IRB approval number:AF-SOP-07-1.1-01). Informed consent was obtained from all patients.

\section{Consent for publication}

Written informed consent for publication was obtained from all participants.

\section{Availability of data and material}

All data generated or analysed during this study are included in this published article and its supplementary information files.

\section{Competing interests}

The authors declare no competing financial interests.

\section{Funding}

This study was supported by the grants from the National Natural Science Foundation of China (No. 81372811) and Science and Technology Agency of Liaoning Province (No. 2013225049).

\section{Authors' contributions}

W.G. G. and Z.L. designed experiments,they made the equal contribution to the article.C. B. participated in the conception of the idea. L.C.Y. and C.Y. contributed to the literature search.L.C.G.participated in the conception and coordination. All authors have read and approved the final manuscript for publication.

\section{Acknowledgements}


We thank the department of Breast Surgery of The First Hospital of China Medical University for providing human breast cancer samples. We also thank the College of China Medical University and Genechem Co.,Ltd. (Shanghai ,China) for technical assistance in experiments.

\section{References}

1. Harbeck N, Penault-Llorca F, Cortes J, Gnant M, Houssami N, Poortmans P, et al. Breast cancer. Nature Reviews Disease Primers. 2019;5(1):66.

2. Nielsen TO, Hsu FD, Jensen K, Cheang M, Karaca G, Hu Z, et al. Immunohistochemical and clinical characterization of the basal-like subtype of invasive breast carcinoma. Clin Cancer Res. 2004;10(16):5367-

3. C M Perou, T Srlie, M B Eisen, M van de Rijn, S S Jeffrey, C A Rees, et al. Molecular portraits of human breast tumours. 2000;406(6797):747-52.

4. Millar EKA, Graham PH, O'Toole SA, McNeil CM, Browne L, Morey AL, et al. Prediction of local recurrence, distant metastases, and death after breast-conserving therapy in early-stage invasive breast cancer using a five-biomarker panel. J Clin Oncol.2009;27(28):4701-

5. Metzger O, Sun ZX, Viale G, Price KN, Crivellari D, Snyder RD, et al. Patterns of recurrence and outcome according to breast cancer subtypes in lymph node-negative disease: results from international breast cancer study group trials VIII and IX. J Clin Oncol.2013;31(25):3083-

6. Arvold ND, Taghian AG, Niemierko A, Abi Raad RF, Sreedhara M, Nguyen PL, et al. Age, breast cancer subtype approximation, and local recurrence after breast-conserving therapy. $\mathrm{J}$ Clin Oncol. 2011;29(29):3885-

7. Goldhirsch A, Wood W C, Coates A S, Gelber R D, Thürlimann B, Senn H-J. Strategies for subtypesdealing with the diversity of breast cancer: highlights of the St. Gallen International Expert Consensus on the Primary Therapy of Early Breast Cancer 2011. Annals of oncology.2011;22(8):1736-47区

8. Tsoutsou PG, Vozenin M-C, Durham A-D, Bourhis J. How could breast cancer molecular features contribute to locoregional treatment decision making? Crit Rev Oncol Hematol. 2017;110:43-

9. Sanpaolo P, Barbieri V, Genovesi D. Prognostic value of breast cancer subtypes on breast cancer specific survival, distant metastases and local relapse rates in conservatively managed early stage breast cancer: a retrospective clinical study. Eur J Surg Oncol.2011;37(10):876-

10. A Garca Fernndez, C Chabrera, M Garca Font, M Fraile, J M Lain, S Gnzalez, et al. Mortality and recurrence patterns of breast cancer patients diagnosed under a screening programme versus comparable non-screened breast cancer patients from the same population: analytical survey from 2002 to 2012. Tumor Biol.2014;35(3):1945-

11. Jatoi I, Anderson WF, Jeong J-H, Redmond CK. Breast cancer adjuvant therapy: time to consider its time-dependent effects. J Clin Oncol.2011;29(17):2301- 
12. Albert JM, Gonzalez-Angulo AM, Guray M, Sahin A, Strom EA, Tereffe W,et al. Estrogen/progesterone receptor negativity and HER2 positivity predict locoregional recurrence in patients with T1a,bN0 breast cancer. Int J Radiat Oncol.2010;77(5):1296-

13. Voduc KD, Cheang MCU, Tyldesley S, Gelmon K, Nielsen TO, Kennecke H. Breast cancer subtypes and the risk of local and regional relapse. J Clin Oncol. 2010;28(10):1684-

14. Nguyen PL, Taghian AG, Katz MS, Niemierko A, Raad RFA, Boon WL, et al. Breast cancer subtype approximated by estrogen receptor, progesterone receptor, and HER-2 is associated with local and distant recurrence after breast-conserving therapy. J Clin Oncol .2008;26(18):2373-

15. Gomig THB, Cavalli IJ, Souza RLR, Vieira E, Lucena ACR, Batista M, et al. Quantitative label-free mass spectrometry using contralateral and adjacent breast tissues reveal differentially expressed proteins and their predicted impacts on pathways and cellular functions in breast cancer. Journal of Proteomics. 2019;199:1-14.

16. Gotz S, Garcia-Gomez JM, Terol J.High-throughput functional annotation and data mining with the Blast2GO suite. Nucleic Acids Res. 2008; 36(10): 16.

17. Lv H, Liu L, Zhang Y , Song $T$, Lu J , Chen $X$. Ingenuity pathways analysis of urine metabonomics phenotypes toxicity of gentamicin in multiple organs. Molecular bioSystems.2010;6(10):2056-67.

18. Wiśniewski JR, Zougman A, Nagaraj N, Mann M.Universal sample preparation method for proteome analysis. Nat Methods.2009;6(5): 359-62.

19. Smid M, Wang Y, Klijn JGM, Sieuwerts AM,Zhang Y, Atkins D,et al. Genes associated with breast cancer metastatic to bone. J Clin Oncol. 2006;24(15):2261-7.

20. Lee SJ, Park S, Ahn HK, Yi JH, Cho EY, Sun JM, et al. Implications of bone-only metastases in breast cancer: Favorable preference with excellent outcomes of hormone receptor positive breast cancer. Cancer Res Treat. 2011;43(2):89-95.

21. Smid M, Wang Y, Zhang Y, Sieuwerts A M, Yu J, Klijn JGM,et al. Subtypes of breast cancer show preferential site of relapse. Cancer Res. 2008;68(9):3108-14.

22. Owen S, Sanders AJ, Ye L, Mason MD, Jiang WG. Osteomodulin expression profile in human breast cancer. EUROPEAN JOURNAL OF CANCER. 2013, 49:S197.

23. Jiang Y, Chen M, Nie H, Yuan Y.PD-1 and PD-L1 in cancer immunotherapy: clinical implications and future considerations. Human Vaccines \& Immunotherapeutics. 2019;15(5):1111-22.

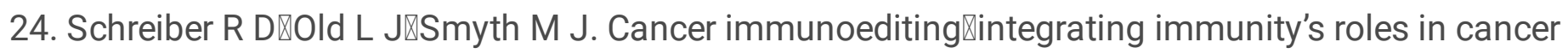
suppression and promotion.2011; 331(6024)凶1565-70.

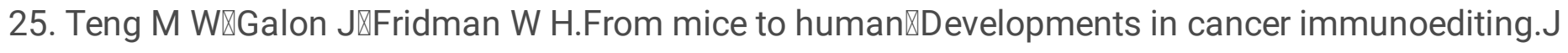
Clin Invest. 2015;125(9):3338-46.

26. Baptista M Z\Saritn L O\Derchain S F®Glauce A P, Jos V.Prognostic significance of PD-L1 and PD-L2 in breast cancer. Hum Pathol. 2016;47(1):78-84.

27. Sobral-Leite M, Van de Vijver K, Michaut M, van der Linden R, Hooijer GKJ, Horlings HM, et al. Assessment of PD-L1 expression across breast cancer molecular subtypes囚in relation to mutation 
rate $\otimes B R C A 1-l i k e$ status $\bigotimes$ tumor-infiltrating immune cells and survival. Oncoimmunology . 2018;7(12):717-23.

28. Ali HR, Glont SE, Blows FM, Provenzano E, Dawson SJ, Liu B, Hiller L,et al. PD-L1 protein expression in breast cancer is rare邓enriched in basal-like tumours and associated with infiltrating lymphocytes.Ann Oncol.2015;26(7):1488-93.

29. Lee J『Kim D MखLee A. Prognostic role and clinical association of tumor-infiltrating lymphocyte区 programmed death ligand-1 expression with neutrophil-lymphocyte ratio in locally advanced triplenegative breast cancer.Cancer Res Treat.2019;51(2):649-63.

30. Muenst S, Schaerli AR, Gao F, Däster S, Trella E, Droeser R A, et al. Expression of programmed death ligand 1ХPD-L1囚is associated with poor prognosis in human breast cancer.Breast Cancer Res Treat. 2014;146(1):15-24.

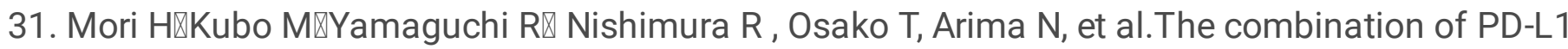
expression and decreased tumor-infiltrating lymphocytes is associated with a poor prognosis in triple-negative breast cancer.2017;8(9):15584-92.

32. Qin T, Zeng YD, Qin G, Xu F, Lu JB, Fang WF, et al. High PD-L1 expression was associated with poor prognosis in 870 Chinese patients with breast cancer. 2015;6(32):33972-81.

33. Robert C, Long GV, Brady B, Dutriaux C, Maio M, Mortier L, et al.Nivolumab in previously untreated melanoma without BRAF mutation. N Engl J Med. 2015;372(4):320-

34. Brahmer J, Reckamp KL, Baas P, Crinò L, Eberhardt WEE,Poddubskaya E,et al. Nivolumab versus docetaxel in advanced squamous-cell non-small-cell lung cancer. N Engl J Med. 2015;373(2):1627.

35. Armand P, Engert A, Younes A, Fanale M, Santoro A, Zinzani PL,et al. Nivolumab for relapsed/refractory classic hodgkin lymphoma after failure of autologous hematopoietic cell transplantation:extended follow-up of the multicohort single-arm phase IICheckMate 205 trial. J Clin Oncol. 2018;36(14):1428-39.

36. El-Khoueiry AB, Sangro B, Yau T, Crocenzi TS, Kudo M, Hsu C, et al. Nivolumab in patients with advanced hepatocellular carcinoma (CheckMate040): an open-label, non-comparative, phase 1/2 dose escalation and expansion trial. 2017;389(10088):2492-502.

37. Peter Burbelo, Anton Wellstein and Richard G Pestell. Pestell.Altered Rho GTPase signaling pathways in breast cancer cells. Breast Cancer Research and Treatment. 2004;84(1): 43-

38. Hui W, Bin W, Qing L , Hongying A, Weidong L , Xuejun J , et al.Overexpression of RhoGDI, a novel predictor of distant metastasis,promotes cell proliferation and migration in hepatocellular carcinoma. FEBS Letters. 2014;588(3): 503-

39. Zhao L, Wang H, Sun X, Ding Y. Comparative proteomic analysis identifies proteins associated with the development and progression of colorectal carcinoma. FEBS J. 2010;277(20):4195-

40. Michael AH, Dan T. RhoGDI signaling provides targets for cancer therapy. European journal of cancer. 2010;46(7):1252-9.

41. Hassan $\mathrm{H}$, Tian X, Inoue $\mathrm{K}$, Chai N, Liu C, Soda K, et al.Essential role of X-box binding protein-1 during endoplasmic reticulum stress in podocytes. J Am Soc Nephrol. 2016;27(4):1055-65. 
42. Iwakoshi NN, Lee AH, Vallabhajosyula P, Otipoby KL, Rajewsky K, Glimcher LH. Plasma cell differentiation and the unfolded protein response intersect at the transcription factor XBP-1. Nat Immunol.2003;4: 321-9.

43. Chen X, Iliopoulos D, Zhang Q, Tang Q, Greenblatt MB,Hatziapostolou M, et al. XBP1 promotes triplenegative breast cancer by controlling the HIF1a Nature.2014;508(7494): 103-7.

44. Bagratuni T, Wu P, Gonzalez de Castro D, Davenport EL,DickensNJ, WalkerBA, et al. XBP1s levels are implicated in the biology and outcome of myeloma mediating different clinical outcomes to thalidomide-based treatments. Blood .2010;116(2):250-3.

45. Patil AS, Sable RB , Kothari RM. An update on transforming growth factor- $\beta(T G F-\beta)$ : Sources, types, functionsand clinical applicability for cartilage/bone healing. J Cell Physiol.2011;226(12):30943103.

46. Liu H, Zhu Y, Zhu H, Cai R, Wang KF, Song J,et al.Role of transforming growth factor $\beta 1$ in the inhibition of gastric cancer cell proliferation by melatonin in vitro and in vivo. Oncology Reports.2019;42(2): 753-62.

47. Muraoka RS, Koh Y, Roebuck LR, Sanders ME, Brantley-Sieders D, Gorska AE,et al. Increased malignancy of Neu-induced mammary tumors overexpressing active transforming growth factor beta 1. Cell Biol. 2003;23:8691.

48. Nam JS, Terabe M, Mamura M, Kang MJ, Chae H, Stuelten C, et al. An anti-transforming growth factor beta antibody suppresses metastasis via cooperative effects on multiple cell compartments. Cancer Res. 2008;68(10):3835-43.

49. Ehata S, Hanyu A, Hayashi M, Aburatani H, Kato Y, Fujime M, et al. Transforming growth factor-beta promotes survival of mammary carcinoma cells through induction of antiapoptotic transcription factor DEC1.Cancer Res. 2007; 67(20): 9694-703.

50. Muraoka RS, Dumont N, Ritter CA, Dugger TC, Brantley DM, Chen J, et al. Blockade of TGF-beta inhibits mammary tumor cell viability, migration, and metastases. Clin. Invest. 2002;109(12):1551-9.

51. Lin RL, Zhao LJ. Mechanistic basis and clinical relevance of the role of transforming growth factor- $\beta$ in cancer. Cancer Biol Med. 2015;12(4):385-

52. Adrian P D, Cheryl J, Tania S, Olivier D.mTOR and Tumor Cachexia. J. Mol. Sci. 2018;19(8): 2225.

53. Hui H, Qingbin K, Hongying Z, Jiao W, Ting L,Yangfu J.Targeting mTOR for cancer therapy. Journal of Hematology \& Oncology .2019;12(1):71.

\section{Figures}



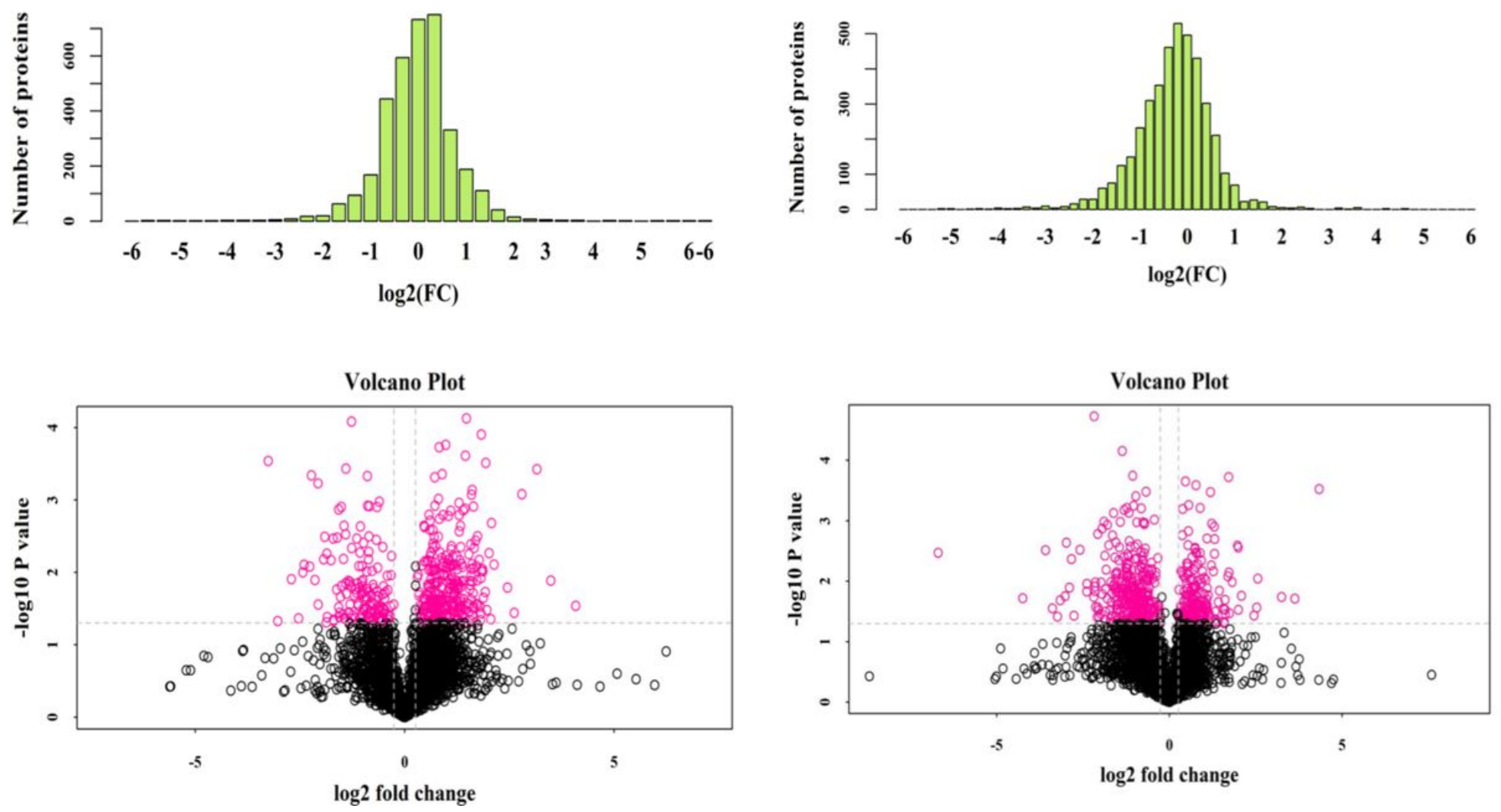

Figure 1

Protein Ratio Distribution and Volcano Map. a.(LA vs PT) and b.(HER2 vs LA). Each comparison of the parallel between the two groups of samples was better. The $x$-coordinate is the multiple of difference (logarithmic transformation based on 2). The ordinate is the number of identified proteins. c.(LA vs PT) and d.(HER2 vs LA). The fold-change and $P$ value of protein expression between the two groups of samples were used to draw the volcano map to show the significant differences between the two groups of sample data. The $x$-coordinate is the difference multiple (log base 2 ) and the $y$-coordinate is the $P$ value (log base 10). 

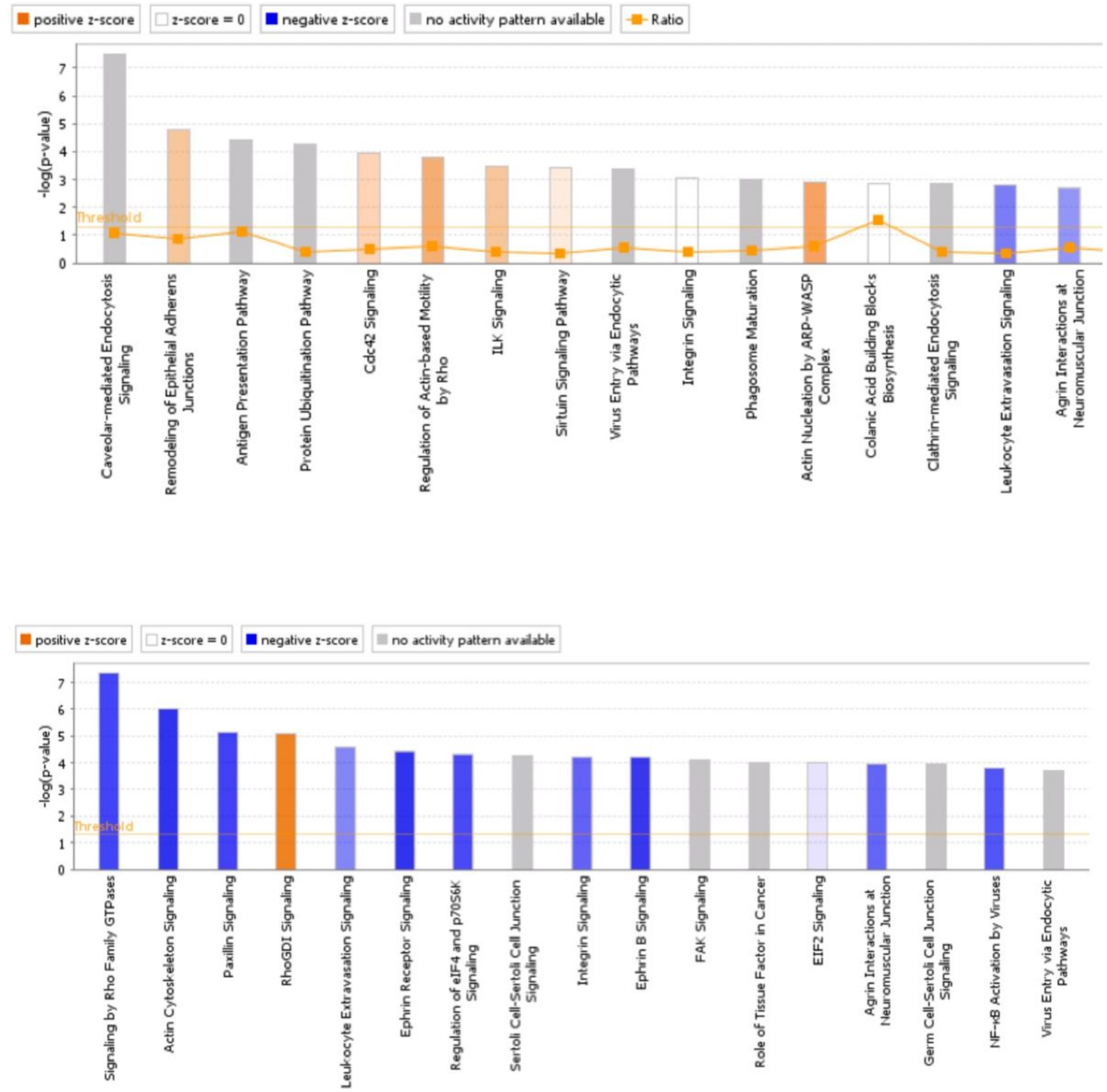

Figure 2

Significant enrichment of DEPs in some classic pathways. a.(LA vs PT) and b.(HER2 vs LA). The xcoordinate is the pathway name, and the $y$-coordinate is the significance level of enrichment. The orange mark indicates that the pathway is activated (z-score $>0)$ and the blue mark indicates that the pathway is inhibited $(z$-score $<0)$. The depth of orange and blue (or the absolute value of the $z$-score) represents the degree of activation or inhibition (according to the internal algorithm and criteria of IPA; a z-score $\geq 2$ means that the pathway is significantly activated and a $z$-score $\leq-2$ means that the pathway is 
significantly suppressed). Ratio represents the ratio of the number of differential genes in the signaling pathway to the number of all genes contained in the pathway.
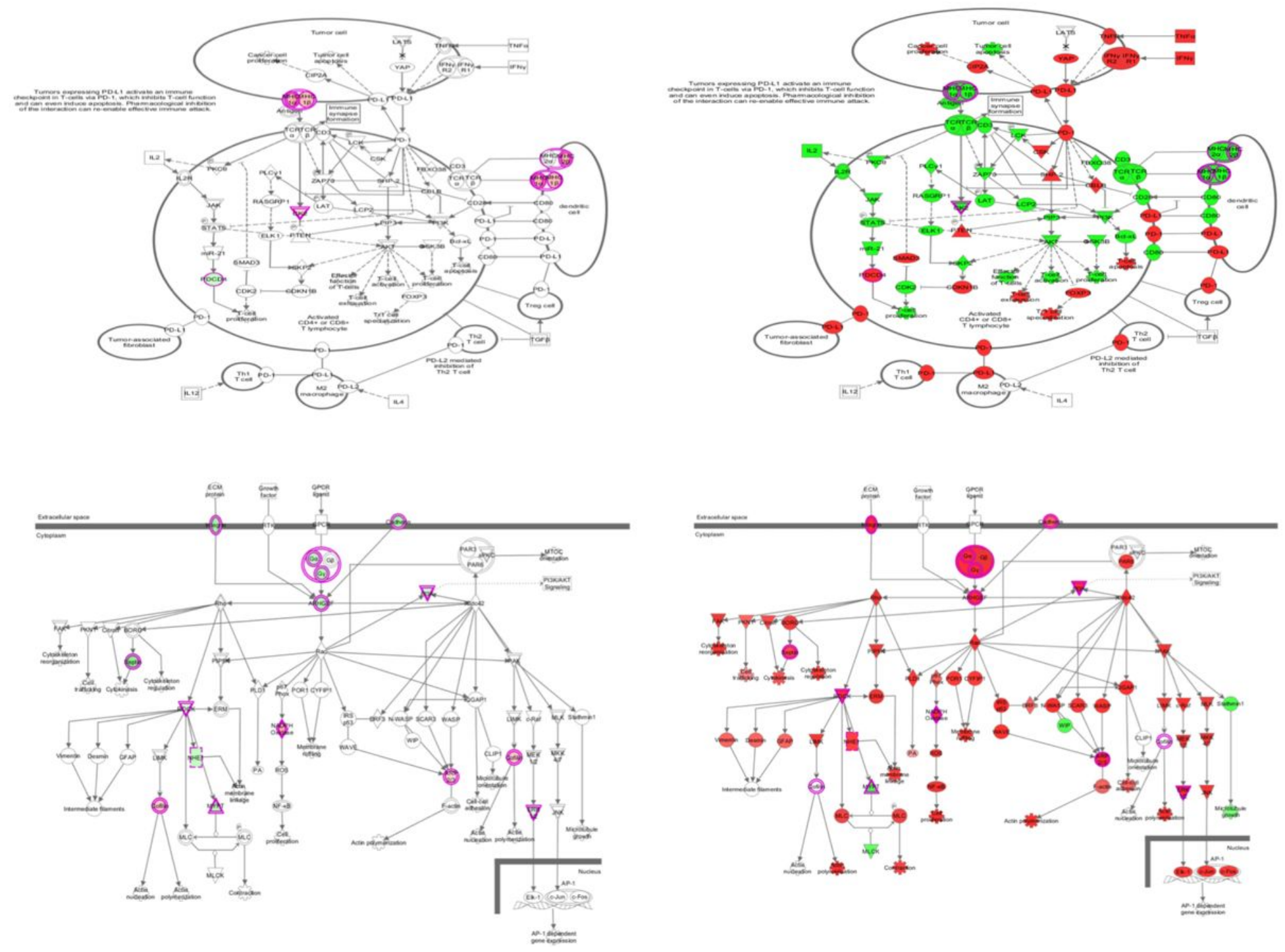

Figure 3

Classic pathway analysis a. (LA vs PT). Expression trend of each protein molecule in the PD-1, PD-L1 cancer immunotherapy pathway inhibition in this study. b. Literature reported the change trend of the expression of various molecules in thePD-1, PD-L1 cancer immunotherapy pathway activation. c . (HER2 vs LA). Expression trend of each protein molecule in the Signaling by Rho Family GTPases inhibition in this study. d. Literature reported the change trend of the expression of various molecules in the Signaling by Rho Family GTPases activation. Red represents up-regulation and green represents down-regulation. 

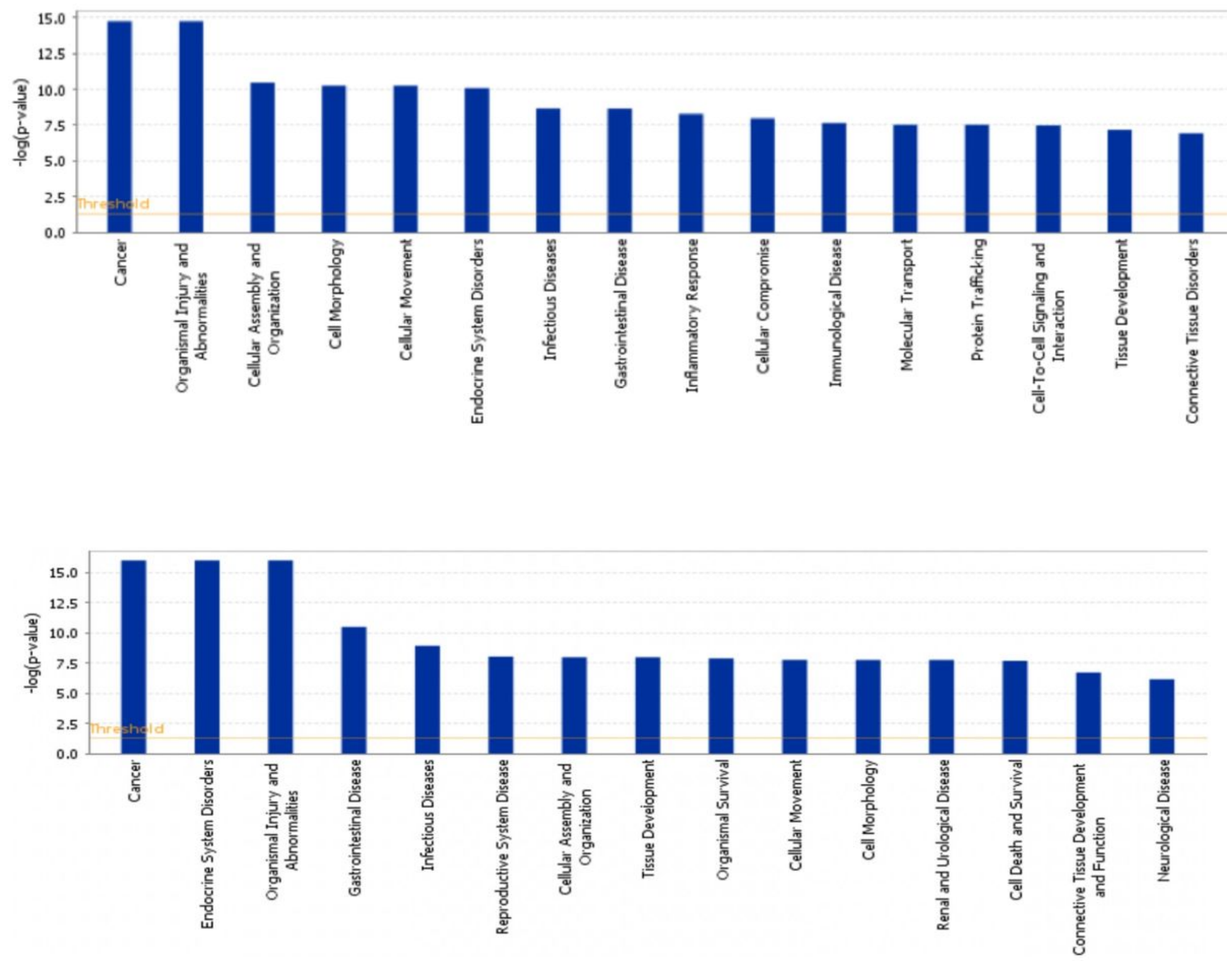

Figure 4

Significant enrichment of DEPs in disease and function. a.(LA vs PT) and b.(HER2 vs LA). The $x-$ coordinate is the pathway name and the $y$-coordinate is the significance level of enrichment (negative logarithmic transformation with base 10). 

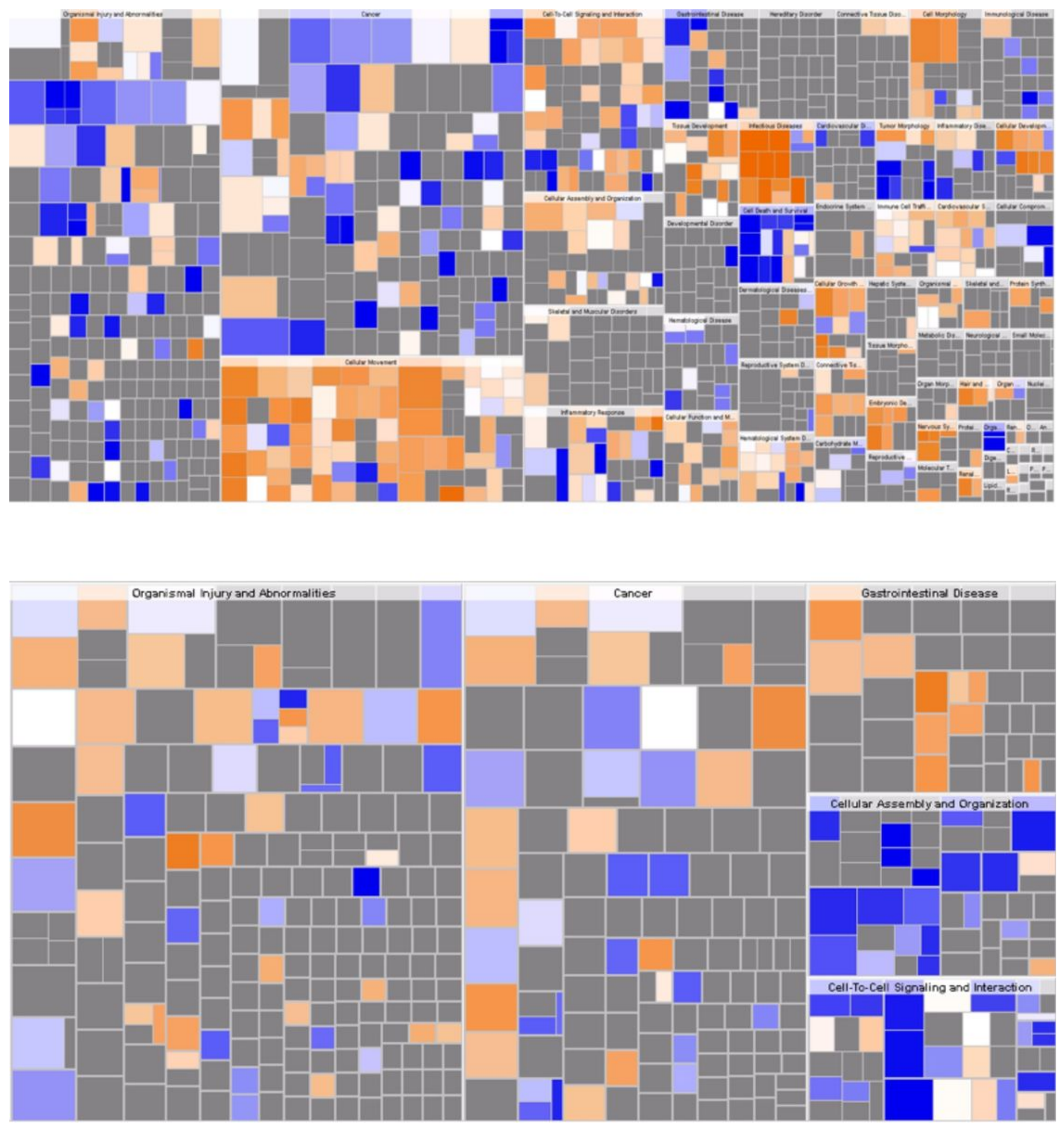

\section{Figure 5}

Disease and functional heat maps. a.(LA vs PT) and b.(HER2 vs LA). Orange represents the activation of disease or functional state (z-score $>0$ ), blue represents the inhibition of disease or functional state ( $z$ score $<0$ ), and gray represents the undetermined disease or functional state (z-score could not be calculated). According to the internal algorithm and criteria of IPA, a z-score $\geq 2$ means that the disease 
or function is significantly activated and a $z$-score $\leq-2$ means that the disease or function is significantly inhibited.
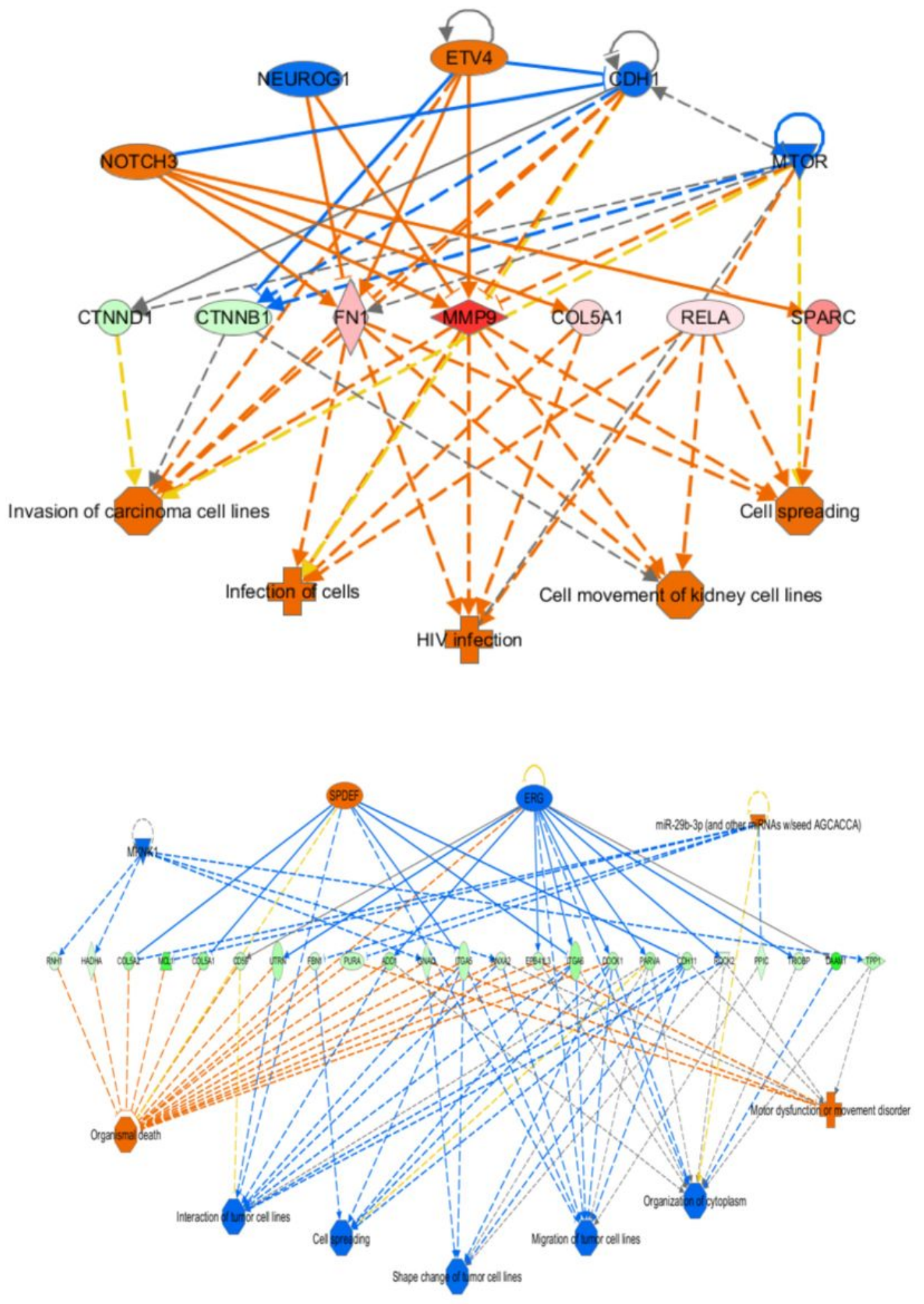

Figure 6

The first regulatory network in Regulation effect analysis. a.(LA vs PT). The data set may be caused by the regulation of $\mathrm{CDH} 1$, ETV4, MTOR, NOTCH3 on Cell movement of kidney Cell lines, Cell spreading, HIV infection, infection of cells through COL5A1, CTNNB1, CTNND1, FN1, MMP9, RELA, SPARC and other 
genes.Invasion of carcinoma cell lines can be activated. b.(HER2 vs LA). The data set may be due to the regulators ERG, mir-29b-3p (and other miRNAs w/seed AGCACCA), MKNK1, SPDEF through ADD1, ANXA2, CD59, CDH11, COL5A1, COL5A2, DAAM1, DOCK1, EPB41L3, FBN1, GNAQ, HADHA, ITGA5, ITGA6,MCL1, PARVA, PPIC, PURA, RNH1, ROCK2, TPP1, TRIOBP, UTRN and other genes have an activation effect on the Organismal death, Motor or movement disorder, while for Cell spreading, Interaction of tumor Cell lines,Migration of tumor cell lines, Organization of cytolines and Shape change of tumor cell lines have inhibitory effects

\section{Supplementary Files}

This is a list of supplementary files associated with this preprint. Click to download.

- supplementarytable4.xlsx

- supplementarytable11.xIsx

- supplementarytable8.xlsx

- supplementarytable10.xIsx

- supplementarytable5.xlsx

- supplementarytable6.xlsx

- supplementarytable9.xlsx

- supplementarytable7.xlsx

- supplementarytable2..xlsx

- supplementarytable3..xlsx

- supplementarytable1..xlsx 